\title{
Computational Analysis on the Role of GPM6A in Human Thyroid Cancer
}

\author{
Zoya Khalid ${ }^{1}$, Sheema Sameen ${ }^{1}$, Shaukat I Malik ${ }^{2 *}$ and Shehzad S
}

${ }^{1}$ Department of Environmental Sciences, International Islamic University, Islamabad, Pakistan

${ }^{2}$ Department of Bioinformatics, Muhammad Ali Jinnah University, Islamabad, Pakistan

\begin{abstract}
Thyroid cancer is one of the major cancers around the world. In this study the whole thyroid genome is systematically scanned in order to hit those molecular targets which are highly associated with thyroid cancer. To achieve this goal bioinformatics methodologies are combined. These include the high throughput microarray analysis combined with Serial analysis of gene expression. The results obtained revealed Glycoprotein M6A (GPM6A) as a novel associated gene marker. It belongs to the glycoprotein's family which plays a major role in cell migration and also known as the major contributors in tumor formation. Moreover the biological pathway of GPM6A is not yet been defined. In this study by assessing the whole biological mechanism the pathway is also inferred. This new drug target will help the biologists in finding the early diagnosis and better treatment for the thyroid cancer patients.
\end{abstract}

Keywords: Thyroid cancer; Microarray; Glycoprotein's; GPM6A

\section{Introduction}

Cancer affects people at all ages with the hazard for most types increasing with age. The proportion of deaths from cancer is expected to rise worldwide [1]. In order to comprehend the biology of cancer it's necessary to dig out the genes which serves as oncogenes. Gene expression analysis is the most gifted field in mining the diseased genes as it provides both the experimental as well as in silico means for screening cancer related genes. Glycoprotein M6A is expressed in neuronal membrane proteins as well as in non-neuronal, but is found to be mainly expressed in neuronal membranes as a stress responsive gene. Stress has an important effect on thyroid autoimmunity. Stress targets endocrine glands including thyroid and lessen the immunity in the body which causes many thyroid diseases [2].

Brain is the key region that response to the environmental stress induced in the body. The neuronal glycoprotein M6A is present in the hippocampus region of the brain by showing its presence by the projection of membrane filopodia. M6A encodes protein which has an important role in synapse and filopodia formation [3]. Stress incites many structural changes in neuronal network predominantly in the hippocampal region. In the hippocampal formation the several genes are involved including CDC like Kinase- 1 and certain G-proteins and the glycoprotein M6A which shows down regulation behavior. Its expression diminishes in chronic stress [4].

The receptor endocytosis function of glycoprotein M6A involves opioid receptors which belongs to the family of G-protein coupled receptors (GPCR) and modulates various mechanisms including stress, mood, appetite and immune responses [5]. It has been noticed that an opiate drug binds to the opioid receptors and stimulates the adaptive changes like tolerance, dependence and addiction. Opiates are considered as the finest drug in treating the stress and chronic pain. The cellular level study specifies that repetitive exposure of these receptors with the drug accelerates the phosphorylation of intracellular domains, which in turn leads to the receptor desensitization and internalization of receptors. This signaling mechanism of opioid receptors is regulated by membrane proteins including glycoprotein M6A [6]. These endogenous opioids stifle the cell proliferation.

Papillary thyroid carcinoma (PTC) is foremost type of thyroid cancer, as more than $80 \%$ of cancers diagnosed are of papillary type [7]. It is a very well differentiated carcinoma and MAPK activation has been associated with PTC. Along with that the RET, NTRK mutation and the point mutations of RAS and BRAF are also linked with it. BRAF V600E mutation has been found as the most frequent cause of PTC [8-10].

Gene expression analysis has known as a tool in mining diseased gene patterns with more ease. In this study major focus is to utilize the bioinformatics approaches to screen more cancer insights for papillary thyroid carcinoma. The Microarray technology has become known as a most promising tool to evaluate the gene patterns. The analysis of these hidden patterns can lead towards enhanced understanding of functional genomics [11-13]. Previously all research made was pure experimental, in order to make the gene expression analysis a bit easier for the researchers, in silico methodology is designed in this study.

\section{Materials and Methods}

The in-silico methodology comprises of two steps, first step involves the microarray analysis and second step involves the verification tools. The difference of two fold among the two phenotypes is considered as remarkable.

\section{Microarray analysis}

For the microarray analysis two datasets GDS1732 and GDS1665 are downloaded from Gene expression omnibus (GEO). The first data set contains the expression profiling of 7 classical papillary thyroid carcinoma (PTC) tissue samples and 7 paired normal thyroid tissue samples (normal group) while the second data set holds classical papillary thyroid carcinoma (PTC) tumors from 9 patients and 9 control samples [14]. On these datasets Multi Experiment viewer software is utilized $[15,16]$.

Statistical tests: These datasets are passed through advanced data

${ }^{*}$ Corresponding author: Department of Bioinformatics, Muhammad Ali Jinnah University, Islamabad, Pakistan, E-mail: msiqbqlpd@yahoo.com

Received October 15, 2011; Accepted January 17, 2012; Published January 20 2012

Citation: Khalid Z, Sameen S, Malik SI, Shehzad S (2012) Computational Analysis on the Role of GPM6A in Human Thyroid Cancer J Data Mining in Genom Proteomics 3:114. doi:10.4172/2153-0602.1000114

Copyright: $\odot 2012$ Khalid Z, et al. This is an open-access article distributed unde the terms of the Creative Commons Attribution License, which permits unrestricted use, distribution, and reproduction in any medium, provided the original author and source are credited. 
Citation: Khalid Z, Sameen S, Malik SI, Shehzad S (2012) Computational Analysis on the Role of GPM6A in Human Thyroid Cancer. J Data Mining in Genom Proteomics 3:114. doi:10.4172/2153-0602.1000114

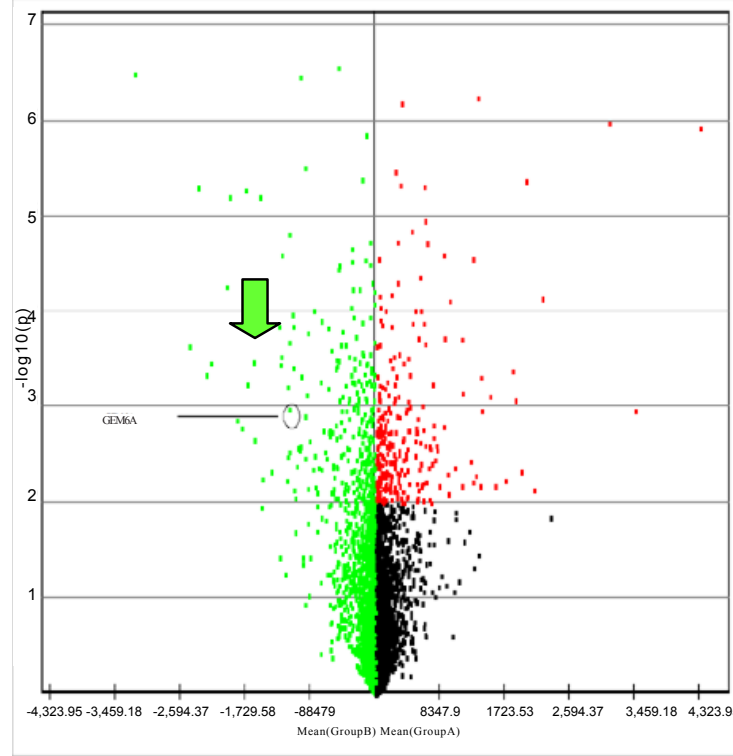

(a)

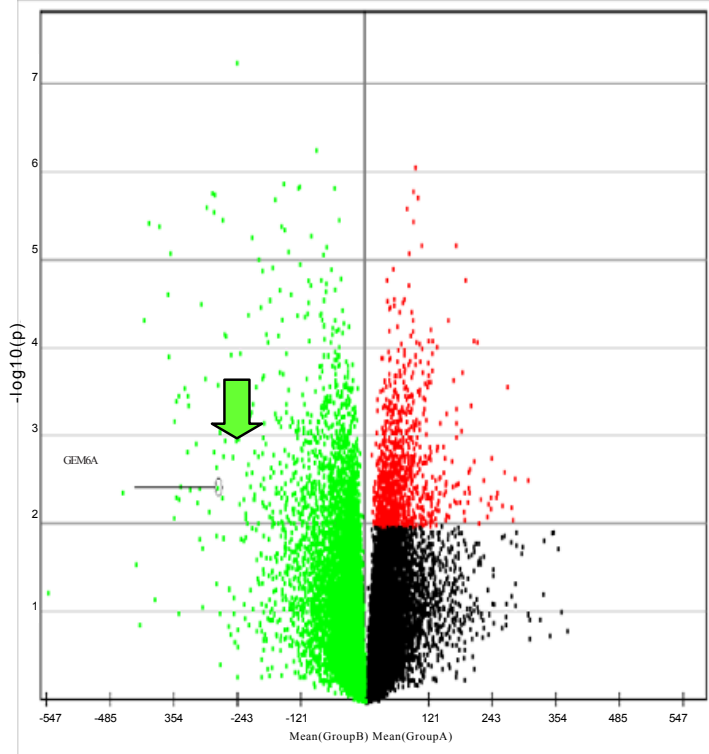

(b)

Figure 1: Volcano plot: P-Value versus mean difference between the two phenotypes. GPM6A is plotted as negative significant gene (a) GDS1732 (b) GDS1665. Red color shows positive significant gene, Green color shows negative significant gene.
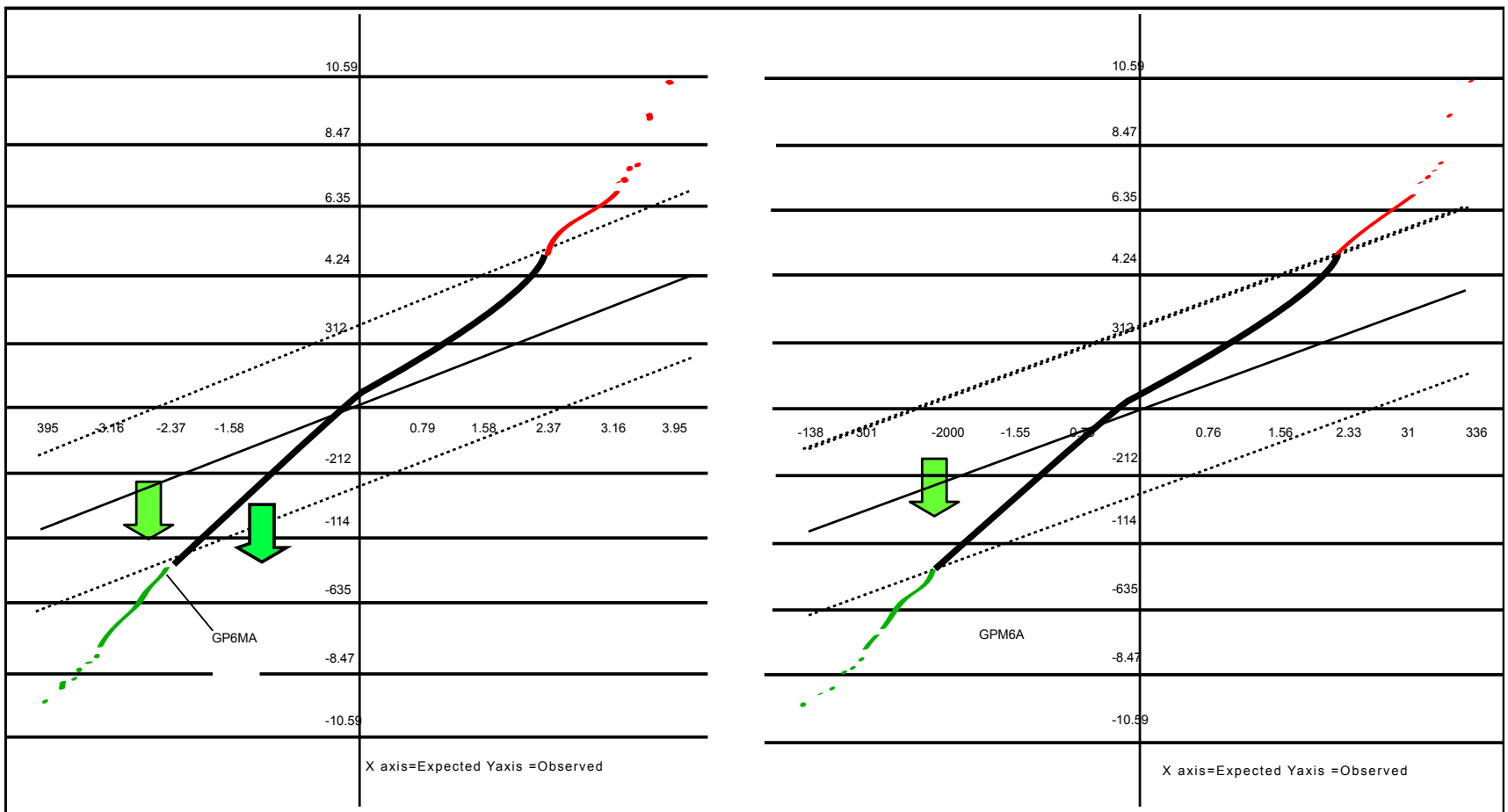

Figure 2: Graph obtained from Significance Analysis of Microarrays. GPM6A as down regulated: negative significant gene (a) GDS1732 (b) GDS1665. Red color shows positive significant gene, Green color shows negative significant gene.

mining approaches which include statistical analysis and the clustering means. The statistical parameters involve T-test and Significance analysis of microarrays (SAM) .The t-test involves the p-value statistics while SAM involves the fold change criteria. These tests divide the gene into two groups significant and non significant. The graphical representation of $t$ test is volcano plot while for the SAM is SAM graph. 
Citation: Khalid Z, Sameen S, Malik SI, Shehzad S (2012) Computational Analysis on the Role of GPM6A in Human Thyroid Cancer. J Data Mining in Genom Proteomics 3:114. doi:10.4172/2153-0602.1000114

The volcano plot is a scatter plot which illustrates the differences of mean expression level and $\log 10 \mathrm{p}$-value, which in turn provides a rapid identification of the changes in huge datasets. In addition SAM divides the genes of the whole datasets into significant group which includes positive significant genes if the mean expression level will be higher in cancer group and negative significant if mean expression level will be higher in normal group. The threshold value for the significant genes is less than $0.005(\mathrm{p}<0.005)$ and fold change should be greater than two fold.

Clustering parameters: Those genes which pass the statistical tests are further employed to the clustering parameters. Three algorithms namely Hierarchical, k-Means Methods and Self organizing tree algorithm (SOTA) are employed. The worth of the clustering techniques depends on the solidity of the results.

\section{Verification tools}

After that for the verification purpose SAGE analysis which verifies the results by computing tags per million counts and the GENT database are utilized. The two datasets U133 plus2 and U133A available at http://medicalgenome.kribb.re.kr/GENT [17] are used. GENT is a web open database. It contains the datasets profiled by Affymetrix Gene Chip U133A or U133plus2. These datasets were collected from public sources. The numbers of tissue samples in both the datasets for cancer phenotype are 106 in total, while for normal thyroid tissue the sample numbers are 50.

\section{Results}

\section{Microarray analysis}

The whole thyroid genome scanning revealed many diseased genes, out of which GPM6A was found as a novel contributor.

Statistical tests: Through the statistical parameters t-test and SAM respective p-value and fold change against GPM6A has been found and listed in table 1 . The graphical representation of t-test: volcano plot and SAM: SAM graph are shown in figures 1 and 2. The graphs plot GPM6A as a down regulator.

Clustering parameters: All of the three clustering means unravel the presence of GPM6A as novel contributor of PTC and a negative significant gene as it shown down regulation behavior clearly highlighted in figures 3, 4, 5 and 6. The difference of expression level between the two phenotypes is greater than two fold which is quite significant. The difference is considered significant only if both of the datasets validates each other's results.

\section{Verification tools}

Furthermore with the SAGE analysis the TPM counts were analyzed which verified the microarray results (Table 2). In addition to that the datasets of GENT also authenticates the results. As in figure 5 and 6 the lower peaks in cancer state or the higher peak in normal phenotype verified the above results. Along with that the biological signaling pathway of GPM6A was also predicted in this study shown in figure 7 .

\begin{tabular}{|c|c|c|c|c|}
\hline Ref_ID & $\begin{array}{l}\text { Gene } \\
\text { Name }\end{array}$ & Description & P-value & Fold Change \\
\hline 209470_s_at & GPM6A & Glycoprotein M6A & $0.001^{* * *} \quad 0.003^{* * *}$ & $>2$ fold \\
\hline
\end{tabular}

Table 1: Respective P-values in GDS1732 and GDS1665 datasets.

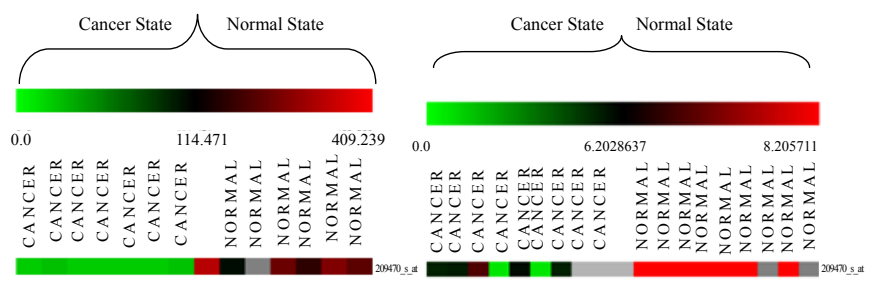

(a)

(b)

Figure 3: Hierarchical Clustering diagrams of the two datasets (a) GDS1732 (b) GDS1665. Red color indicates the highly expressed genes, Green color shows down regulating genes in the datasets.
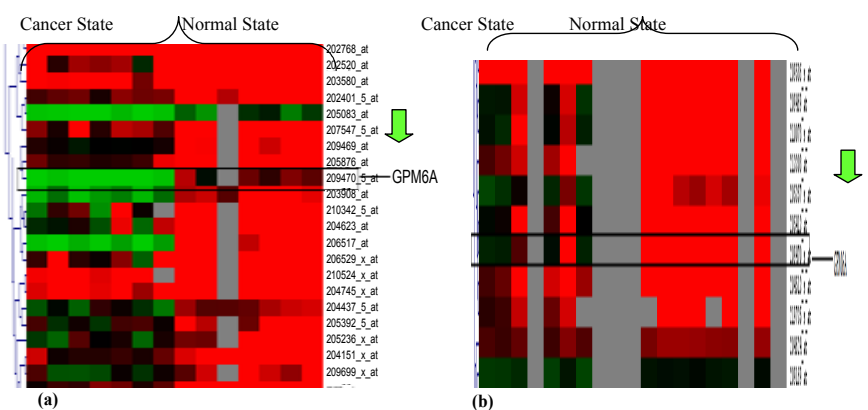

Figure 4: K-Means Clustering diagrams of the two datasets (a) GDS1732 (b) GDS1665. Red color indicates the highly expressed genes, Green color shows down regulating genes in the datasets.

(a)
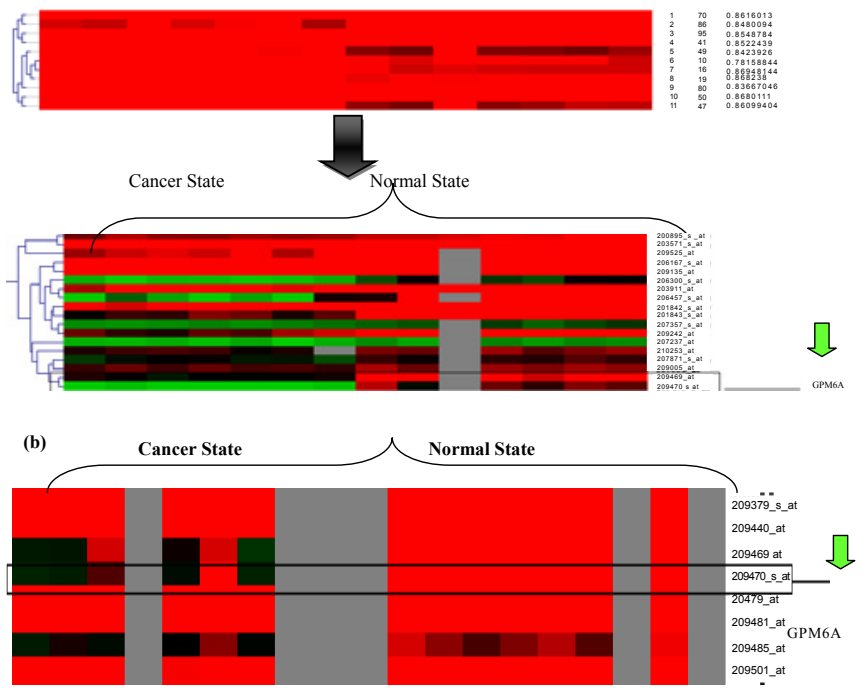

Figure 5: Self Organizing Tree Algorithm diagrams of the two datasets (a) GDS1732 (b) GDS1665. Red color indicates the highly expressed genes, Green color shows down regulating genes in the datasets

\section{Discussion}

Thyroid cancer accounts as one of the major cancers around the world. It is burgeoning as a main health load for the society; its diverse behavior is serving as a continuous mystery for the researchers. There is a requirement of proficient strategies for the early diagnosis and better 
Citation: Khalid Z, Sameen S, Malik SI, Shehzad S (2012) Computational Analysis on the Role of GPM6A in Human Thyroid Cancer. J Data Mining in Genom Proteomics 3:114. doi:10.4172/2153-0602.1000114

(a)

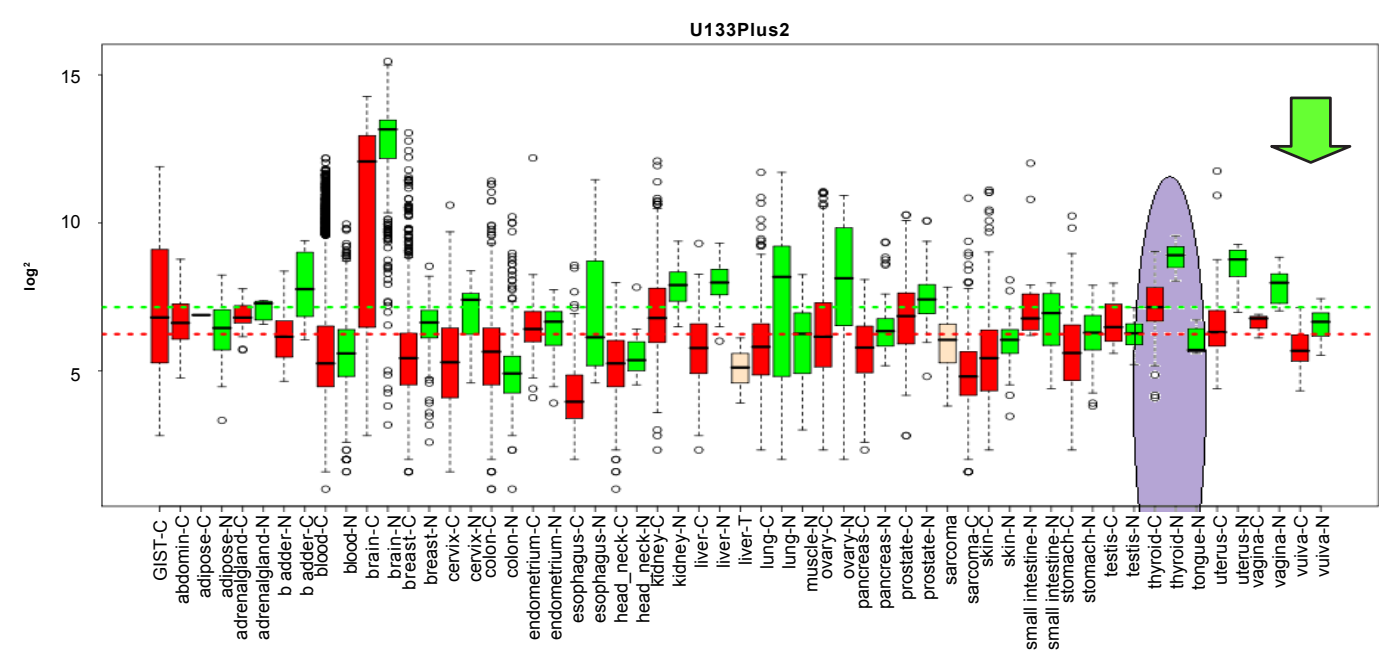

C: cancer

N: Normal

(b)

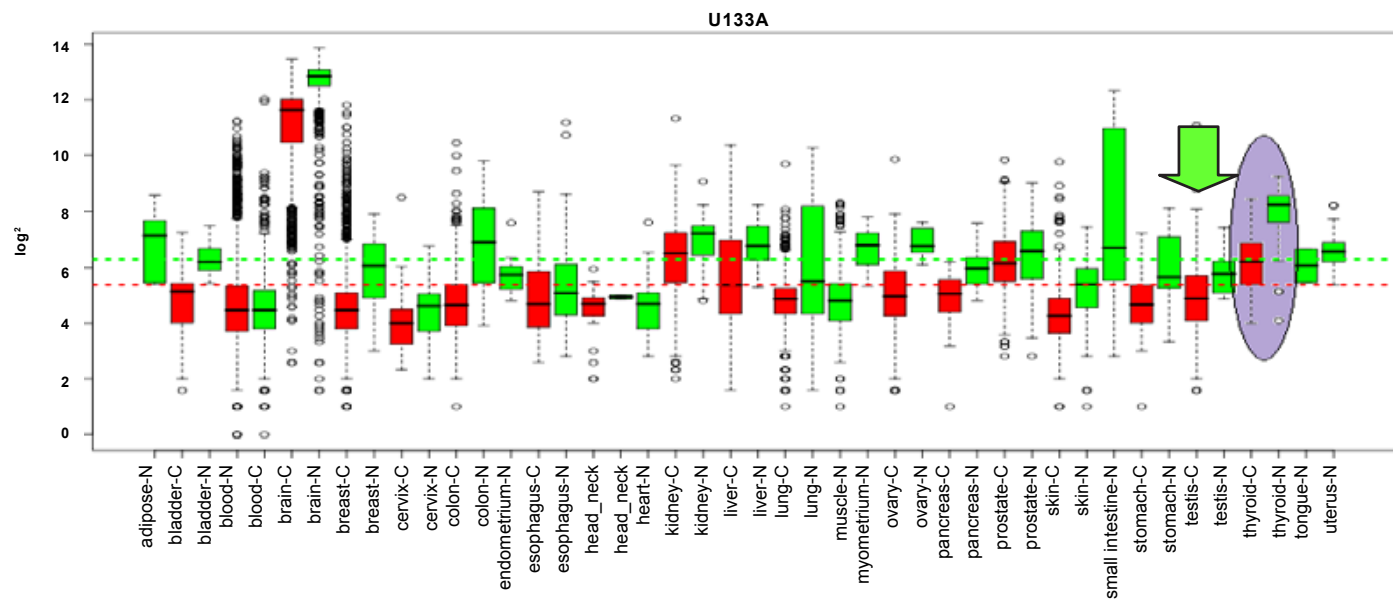

Y-axis: Normalized range of frequency.

X-axis: Cancer types

Figure 6: The difference of expression level shows the down expression of GPM6A in U133Plus 2 and U133A.

treatment. In the past few years a lot of effort has been made in order to solve this secrecy but still there is a need of more work.

With the help of this methodology a novel associated member GPM6A is predicted. From the microarray analysis which first includes the statistical analysis, both the parameters illustrated it as a significant gene. P-value obtained 0.001 and 0.003 is quite significant affirmed this gene as a negative regulator. Likewise SAM plots GPM6A as a negative significant gene. Each illustrated cluster stated the down regulating expression of GPM6A. Additionally SAGE and the microarray datasets of GENT and Array express also confirmed this result.

The entire scanning of thyroid genome revealed some other major contributors like HGD (Homogentisate 1,2- dioxygenase), SLC4A4 (Solute carrier family 4,sodium bicarbonate co transporter member 4), CDH16 (cadherin 16). These are found to be down regulated in papillary thyroid cancer as reported [17-19].

Glycoprotein M6A shows homology with the proteolipid protein family (PLP) restricted to the axonal membranes. The M6A glycoprotein was involved in various biological processes which include neuronal remodeling, Ca ions transportation and trafficking of mu-opoid receptors and it also plays a major role in G-protein coupled receptors endocytosis and intracellular trafficking [20] . M6A shows neuronal and non-neuronal type of expressions. Neuronal M6A is expressed by neurons located in the central nervous system while limited non-neuronal expression is seized to the apical membranes of epithelial cells from early developmental stage. Further, the stress induced changes in the structure of M6A is the hot issue [21]. As the M6A is localized in plasma membrane, this makes M6A significant in the filopodia formation and Src and MAPK kinases contributes in M6A signal propagation [22].

As it is known that cell migration is important for tissue repair and regeneration, immune surveillance and embryonic morphogenesis; unusual cell movement will lead to many diseases including cancer progression. Cell movement is a process which is instigated by the projection of cell membrane formed by filopodia which depends on the structure and function [23]. Filopodia formation is vital for many cellular processes which most notably include development, immunity, angiogenesis, wound healing and metastasis [24]. Cell migration is also 
an important stair in tumor development and progression. Cancer cells travel within the tissues by their own motility which in turn has no control in the cell movement causing the tumor to form. As glycoprotein M6A involved in filopodia formation, that further assists in cell migration [25].

It has been found that GPM6A has already been reported with colorectal and Breast cancer [26,27]. Its expression behavior is down regulating in cold thyroid nodules (CTNs) as reported [28]. GPM6A is not yet found with thyroid cancer. This study shows that GPM6A is involved in papillary thyroid cancer as down expressed gene.

Moreover the biological pathway of GPM6A is not yet been defined. In this study by assessing the whole biological mechanism the pathway is inferred. GPM6A binds with two more proteins CDC like kinase-1 and G-protein in the hippocampus region of the brain. GPM6A responds to stress conditions which effect either directly or indirectly on the immune system involving endocrine or nervous system. The GPM6A is involved in filopodia formation in cell which has an important role in cell migration. As stress induced, the GPM6A shows down regulation expression, that in turn has effect on the filopodia formation and finally on the cell migration. This all leads to cell proliferation (Figure 7). Additionally the opioid drugs acts as treatment for stress, they binds with the receptors present on the membrane, this signaling cascade is modulated by GPM6A. The obstruction in opoid receptors will lead to tumor progression.

In this study the datasets were not only downloaded from one source, they were utilized from two different sources one is GEO and other is GENT to make the data larger in amount which further helps in verification of the results. Furthermore the clustering parameters chosen works best for gene expression datasets. The use of more than one clustering method in gene expression analysis facilitates in providing the best and confident way out.

The worth of the clustering techniques depends on the constancy of the results. This designed in silico scheme can provide the best possible ways to observe the gene expression analysis; it provides the list of steps to be taken in order to analyze the gene expression in the premium mode.

New challenges occur when dealing with multifaceted biological

\begin{tabular}{|l|l|l|l|l|}
\hline \multirow{2}{*}{ Gene ID } & \multicolumn{3}{|l|}{ SAGE Anatomic Viewer } & Virtual Northern Blot Analysis \\
\cline { 2 - 5 } & SAGE tags & Unigene Clusters & Normal (TPM) & Cancer (TPM) \\
\hline GPM6A & ATTATGCATT & Hs.728134 & 2 & 0 \\
\hline
\end{tabular}

Table 2: SAGE Anatomic Viewer and Virtual Northern Blot Analysis.
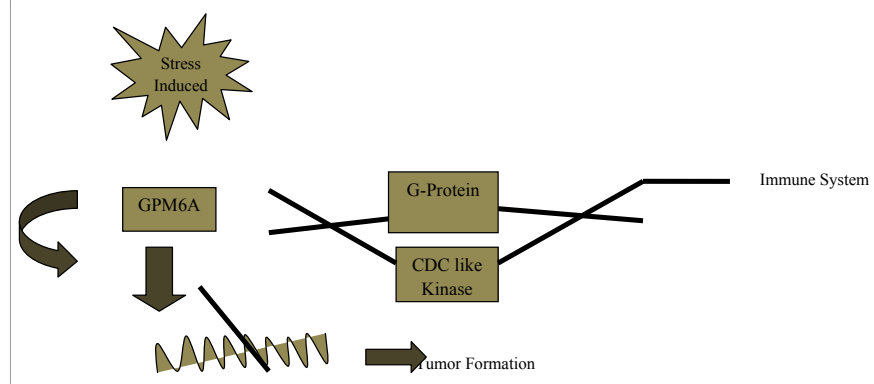

Figure 7: Novel predicted pathway. When the stress is induced in the body the GPM6A becomes down regulated, which in turn stops filopodia formation and leads towards cancer formation. pathways. This predicted marker will help in unveiling the molecular events of tumorigenesis. Additional explorations are required to provide mechanistic insights of theis predicted gene in thyroid cancer by probing large datasets as well as by performing their in vitro and in vivo analysis.

\section{References}

1. Vogelstein B, Kinzler KW (1993) The multistep nature of cancer. Trends Genet 9: 138-141.

2. Cremaschi GA, Gorelik G, Klecha AJ, Lysionek AE, Genaro AM (2000) Chronic stress influences the immune system through the thyroid axis. Life Sci 67 3171-3179.

3. Alfonso J, Fernández ME, Cooper B, Flugge G, Frasch AC (2005) The stressregulated protein $\mathrm{M} 6 \mathrm{a}$ is a key modulator for neurite outgrowth and filopodium/ spine formation. Proc Natl Acad Sci U S A 102: 17196-17201.

4. Cooper B, Fuchs E, Flügge G (2009) Expression of the axonal membrane glycoprotein M6a is regulated by chronic stress. PLoS One 4: e3659.

5. Arstikaitis P, Gauthier-Campbell C, Huang K, El-Husseini A, Murphy TH (2011) Proteins that promote filopodia stability, but not number, lead to more axonaldendritic contacts. PLoS One 6: e16998.

6. Alfaras-Melainis K, Gomes I, Rozenfeld R, Zachariou V, Devi L (2009) Modulation of opioid receptor function by protein-protein interactions. Front Biosci 14: 3594-3607.

7. Wu DF, Koch T, Liang YJ, Stumm R, Schulz S, et al. (2007) Membrane glycoprotein $\mathrm{M} 6 \mathrm{a}$ interacts with the micro-opioid receptor and facilitates receptor endocytosis and recycling. J Biol Chem 282: 22239-22247.

8. Kim HS, Kim do H, Kim JY, Jeoung NH, Lee IK, et al. (2010) Microarray analysis of papillary thyroid cancers in Korean. Korean J Intern Med 25: 399-407.

9. DeLellis RA (2006) Pathology and genetics of thyroid carcinoma. J Surg Onco 94: 662-669.

10. Zagon IS, McLaughlin PJ (1989) Opioid antagonist modulation of murine neuroblastoma: a profile of cell proliferation and opioid peptides and receptors. Brain Res 482: 16-28.

11. Nikiforova MN, Kimura ET, Gandhi M, Biddinger PW, Knauf JA, et al. (2003) BRAF mutations in thyroid tumors are restricted to papillary carcinomas and anaplastic or poorly differentiated carcinomas arising from papillary carcinomas. $\mathrm{J}$ Clin Endocrinol Metab 88: 5399-5404.

12. Pauws E, Veenboer GJ, Smit JW, de Vijlder JJ, Morreau H, et al. (2004) Genes differentially expressed in thyroid carcinoma identified by comparison of SAGE expression profiles. FASEB J 18: 560-561.

13. Allison DB, Cui X, Page GP, Sabripour M (2006) Microarray data analysis: from disarray to consolidation and consensus. Nat Rev Genet 7: 55-65.

14. Lee NH, Saeed Al (2007) Microarrays: an overview. Methods Mol Biol 353 265-300.

15. Slonim DK (2002) From patterns to pathways: gene expression data analysis comes of age. Nat Genet 32: 502-508.

16. He H, Jazdzewski K, Li W, Liyanarachchi S, Nagy R, et al. (2005) The role of microRNA genes in papillary thyroid carcinoma. Proc Natl Acad Sci U S A 102 19075-19080.

17. Calì G, Gentile F, Mogavero S, Pallante P, Nitsch R, et al. (2011) CDH16/KspCadherin Is Expressed in the Developing Thyroid Gland and Is Strongly DownRegulated in Thyroid Carcinomas. Endocrinology 153: 522-534.

18. Fujarewicz K, Jarzab M, Eszlinger M, Krohn K, Paschke R, et al. (2007) A multigene approach to differentiate papillary thyroid carcinoma from benign lesions: gene selection using support vector machines with bootstrapping. Endocr Relat Cancer 14: 809-826.

19. Gałeza-Kulik M, Zebracka J, Szpak-Ulczok S, Czarniecka AK, Kukulska A, et al. (2006) Expression of selected genes involved in transport of ions in papillary thyroid carcinoma. Endokrynol Pol 56: 26-31.

20. Saeed Al, Sharov V, White J, Li J, Liang W, et al. (2003) TM4: a free, opensource system for microarray data management and analysis. Biotechniques 34: $374-378$ 
Citation: Khalid Z, Sameen S, Malik SI, Shehzad S (2012) Computational Analysis on the Role of GPM6A in Human Thyroid Cancer. J Data Mining in Genom Proteomics 3:114. doi:10.4172/2153-0602.1000114

21. Saeed Al, Bhagabati NK, Braisted JC, Liang W, Sharov V, et al. (2006) TM4 microarray software suite. Methods Enzymol 411: 134-193.

22. Shin G, Kang TW, Yang S, Baek SJ, Jeong YS, et al. (2011) GENT: gene expression database of normal and tumor tissues. Cancer Inform 10: 149-157.

23. Cooper B, Fuchs E, Flügge G (2009) Expression of the axonal membrane glycoprotein M6a is regulated by chronic stress. PLoS One 4: e3659.

24. Scorticati C, Formoso K, Frasch AC (2011) Neuronal glycoprotein M6a induces filopodia formation via association with cholesterol-rich lipid rafts. J Neurochem 119: 521-531.

25. Yamaguchi $\mathrm{H}$, Condeelis $\mathrm{J}$ (2007) Regulation of the actin cytoskeleton in cancer cell migration and invasion. Biochim Biophys Acta 1773: 642-652.
26. Applewhite DA, Barzik M, Kojima S, Svitkina TM, Gertler FB, et al. (2007) Ena/VASP proteins have an anti-capping independent function in filopodia formation. Mol Biol Cell 18: 2579-2591.

27. Yamazaki D, Kurisu S, Takenawa T (2005) Regulation of cancer cell motility through actin reorganization. Cancer Sci 96: 379-386.

28. Camps J, Nguyen QT, Padilla-Nash HM, Knutsen T, McNeil NE, et al. (2009) Integrative genomics reveals mechanisms of copy number alterations responsible for transcriptional deregulation in colorectal cancer. Genes Chromosomes Cancer 48: 1002-1017.

29. Eszlinger M, Wiench M, Jarzab B, Krohn K, Beck M, et al. (2006) Meta- and reanalysis of gene expression profiles of hot and cold thyroid nodules and papillary thyroid carcinoma for gene groups. J Clin Endocrinol Metab 91: 19341942. 